\title{
Proving Security Against Chosen Ciphertext Attacks
}

\author{
Manuel Blum* \\ Computer Science Dept. \\ Univ. of Calif. \\ Berkely, CA
}

\author{
Paul Feldman \\ MIT Lab. for Computer Sci \\ Cambridge, MA
}

\author{
Silvio Micali ${ }^{\dagger}$ \\ Lab. for Computer Science \\ MIT \\ Cambridge, MA
}

\begin{abstract}
The relevance of zero knowledge to cryptography has become apparent in the recent years. In this paper we advance this theory by showing that interaction in any zero-knowledge proof can be replaced by sharing a common, short, random string. This advance finds immediate application in the construction of the first public-key cryptosystem secure against chosen ciphertext attack.

Our solution, though not yet practical, is of theoretical significance, since the existence of cryptosystems secure against chosen ciphertext attack has been a famous long-standing open problem in the field.
\end{abstract}

\section{Introduction}

Recently [GMR] have shown that it is possible to prove that some theorems are true without giving the slightest hint of why this is so. This is rigorously formalized in the somewhat paradoxical notion of a zero-knowledge proof system.

If secure encryption schemes exist, though, these proof systems are far from being a rare and bizar event. In fact, under this assumption, [GMW] demonstrate that any language in NP possesses zero-knowledge proof systems.

"Supported by NSF Grant \# DCR85-13926

†Supported by NSF grant \# CCR-8719689 
Actually, as recently pointed out by Impagliazzo [I] and Ben-Or, Goldreich, Goldwasser, Hastad, Kilian, Micali and Rogaway [BGGHKMR], the same is true for all languages in IP; also, as pointed out by Blum [B2], any theorem at all admits a proof that conveys zero-knowledge other than betraying its own length.

Zero-knowledge proofs have proven very useful both in complexity theory and in cryptography. For instance, in complexity theory, via results of fortnow $[F]$ and Boppana and Hastad [BH], zero-knowledge provides us an avenue to convince ourselves that certain languages are not NP-complete. In cryptography, zero-knowledge proofs have played a major role in the recently proven completeness theorem for protocols with honest majority [GMW2]. They also have inspired rigorously-analyzed identification schemes that are as efficient [FFS] and even more efficient [MS] than folklore ones.

Despite its wide applicability, zero-knowledge remains an intriguing notion: What makes zero-knowledge proofs work?

Three main features differentiate all known zero-knowledge proof systems from more traditional ones:

1. Interaction: The prover and the verifier talk back and forth

2. Hidden Randomization: The verifier tosses coins that are hidden from the prover and thus unpredictable to him.

3. Computational Difficulty: The prover imbeds in his proofs the computational difficulty of some other problem.

At a first glance, all of these ingredients appear to be necessary. This paper makes a first, important step in distilling what is essential in a zero-knowledge proof. We show that computational difficulty alone (for instance the hardness of distinguishing products of 2 primes from products of 3 primes) may make inessential the first resource (interaction) and and eliminate the secrecy of the second resource (randomness). That is, if the prover and the verifier share a common random string, the prover can non-interactively and yet in zero-knowledge convince the verifier of the validity of any theorem he may discover. A bit more precisely, for any constants $c$ and $d$, sharing a $k$-bit long random string allows a prover $\mathrm{P}$ to prove in zero-knowledge to a poly $(\mathrm{k})$-time verifier $V$ any $k^{c}$ theorems of $k^{d}$ size non-interactively; that is, without ever reading any message $i$ from $V$.

A Conceptual Scenario: Think of $P$ and $V$ as two mathematicians. After having played "heads and tails" for a while, or having both witnessed the same random event, $P$ leaves for a long trip along the world, during which he continues his mathematical investigations. whenever he discovers a theorem, he writes a postcard to $\mathrm{v}$ proving the validity of his new theorem in zero-knowledge. Notice that this is necessarily a non-interactive process; better said, it is a mono-directional interaction: From $P$ to $V$ only. in fact, even if $V$ would like to answer or talk to $P$, he couldn't: $P$ has no fixed (or predictable) address and will move away before any mail can reach him. 


\subsection{Our Model Versus the Old One}

While the definition of zero-knowledge remains unchanged, the mechanics of the computation of the prover and verifier changes dramatically.

Notice that sharing a random string $\sigma$ is a weaker requirement than being able to interact. In fact, if $\mathrm{P}$ and $\mathrm{V}$ could interact they would be able to construct a common random string by coin tossing over the phone [B1]; the converse, however, is not true.

Also notice that sharing a common random string is a requirement even weaker than having both parties access a random beacon in the rabin's sense (e.g. - perhaps! the same geiger counter). In this latter case, in fact, all made coin tosses would be seen by the prover, but the future ones would still be unpredictable to him. by contrast, our model allows the prover to see in advance all the coin tosses of the verifier. That is the zero-knowledgeness of our proofs does not depend on the secrecy, or unpredictability of $\sigma$, but on the "well mixedness" of its bits! This curious property makes our result potentially applicable. For instance, all libraries in the country possess identical copies of the random tables prepared by the rand corporation. Thus, we may think of ourselves as being already in the scenario needed for non-interactive zero-knowledge proofs.

\subsection{The Robustness of Our Result}

As we have already said, we guarantee that all theorems proved in our proof systems are correct and zero-knowledge if the string $\sigma$ is a truly random one. We may rightly ask what would happen if $\sigma$ was not, in fact, truly randomly selected. fortunately, the poor randomness of $\sigma$ may upset the zero-knowledgeness of our theorems, but not their correctness. That is, for almost all (poorly random) $\sigma$ 's, there is no wrong statement that can be accepted by the verifier. This is indeed an important property as we can never be sure of the quality of our natural sources of randomness. Unfortunately, due to the limitations of an extended abstract, we cannot further elaborate on this and similar points. We wish, however, to point out the following important corollary of our result.

\subsection{Applications of our Result}

A very noticeable application of non-interactive zero-knowledge is the construction of encryption schemes á la Diffie and Hellman that are secure against chosen ciphertext attacks. Whether such schemes existed has been a fundamenatal open problem ever since the appearence of complexity-based cryptography. We will discuss this application in Section 3.

\subsection{What's Coming}

The next section is devoted to set up our notation, recall some elementary facts from Number Theory and state the complexity assumption which sufficies to show the existence of non-interactive, zero-knowledge proofs. 
In Section 3, we show the "single-theorem" case. That is, we show that if a $k^{4}$-bit string $\sigma$ is randomly selected and given to both the proven and the verifier, then the first can prove, for any single string $x$ (of length $k$ ) belonging to a NP-language $L$, that indeed $x \in L$; the proof will be a zero-knowledge one for whenever $\mathrm{x}$ is independent of $\sigma$.

In the final paper [BDFMP], we will show the "many-theorems" case. Namely, that for each fixed polynomial $Q(\cdot)$, using the same randomly chosen $k^{4}$-bit string, the prover can show in zero-knowledge membership in NP languages for any $Q(k)$ strings of length $Q(k)$.

The complexity assumption under which the result holds is the computational difficulty of deciding quadratic residuosity.

We would like to point out that the proof of the many-theorems result in the earlier versions of [BFM] and [DMP] contained a gap: it required, over than the stated number theoretic assumptions, a stronger property about pseudo-random generators. This stronger property is not needed in the final paper.

\section{Preliminaries}

\subsection{Notations and Conventions}

Let us quickly recall the standard notation of [GoMiRi].

We emphasize the number of inputs received by an algorithm as follows. If algorithm a receives only one input we write " $A(\cdot)^{n}$, if it receives two inputs we write " $A(\cdot, \cdot)$ " and so on.

If $A(\cdot)$ is a probabilistic algorithm, then for any input $x$, the notation $A(x)$ refers to the probability space that assigns to the string $\sigma$ the probability that $A$, on input $x$, outputs $\sigma$. If $S$ is a probability space, then $P R_{S}(e)$ denotes the probability that $S$ associates with the element $e$.

If $f(\cdot)$ and $g(\cdot, \ldots, \cdot)$ are probabilistic algorithms then $f(g(\cdot, \ldots, \cdot))$ is the probabilistic algorithm obtained by composing $f$ and $g$ (i.e. running $f$ on $g$ 's output). For any inputs $x, y, \ldots$ the associated probability space is denoted by $f(g(x, y, \ldots))$.

If $s$ is any probability space, then $x \leftarrow S$ denotes the algorithm which assigns to $x$ an element randomly selected according to $S$. If $f$ is a finite set, then the notation $x \leftarrow f$ denotes the algorithm which assigns to $x$ an element selected according to the probability space whose sample space is $f$ and uniform probability distribution on the sample points.

The notation $\operatorname{Pr}(x \leftarrow S ; y \leftarrow T ; \ldots: p(x, y, .)$.$) denotes the probability that the$ predicate $p(x, y, \ldots)$ will be true after the ordered execution of the algorithms $x \leftarrow$ $S, y \leftarrow T, \ldots$

The notation $\{x \leftarrow S ; y \leftarrow T ; \ldots:(x, y, \ldots)\}$ denotes the probability space over $\{(x, y, \ldots)\}$ generated by the ordered execution of the algorithms $x \leftarrow S, y \leftarrow T, \ldots$

Let us recall the basic definitions of [GMR]. We address the reader to the original paper for motivation, interpretation and justification of these definitions. 
Let $U=\{U(x)\}$ be a family of random variables taking values in $\{0,1\}^{*}$, with the parameter $x$ ranging in $\{0,1\}^{*} . U=\{U(x)\}$ is called poly-bounded family of random variables, if, for some constant $e \in \backslash$, all random variables $U(x) \in u$ assign positive probability only to strings whose length is exactly $|x|^{e}$.

Let $C=\left\{C_{x}\right\}$ be a poly-size family of boolean circuits, that is, for some constants $c, d>0$, all $C_{x}$ have one boolean output and at most $|x|^{c}$ gates and $|x|^{d}$ inputs. In the following, when we say that a random string, chosen according to $U(x)$, where $\{U(x)\}$ is a poly-bounded family of random variables, is given as input to $C_{x}$, we assume that the length of the strings that are assigned positive probability by $U(x)$ equals the number of boolean inputs of $C_{x}$.

Definition 2.1 (Indistinguishability) . Let $L \subset\{0,1\}^{*}$ be a language. Two polybounded families of random variables $U=\{U(x)\}$ and $V=\{V(x)\}$ are indistinguishable on $L$ if for all poly-size families of circuits $C=\left\{C_{x}\right\}$,

$$
\begin{gathered}
\mid \operatorname{Pr}\left(A \leftarrow U(x): C_{x}(a)=1\right)- \\
\left.\operatorname{Pr}\left(a \leftarrow V(x): C_{x}(a)=1\right)|<| x\right|^{\circ}
\end{gathered}
$$

For all positive constants $c$ and sufficiently large $x \in L$.

Definition 2.2 (Approximability). Let $L \subset\{0,1\}^{*}$ be a language. a family of random variables $U=\{U(x)\}$ is approximable on $L$ if there exists a probabilistic turing machine $M$, running in expected polynomial time, such that the families $\{U(x)\}$ and $\{M(x)\}$ are indistinguishable on $L$.

\subsection{Number Theory}

Let $Z_{s}(k)$ denote the set of integers product of $s \geq 1$ distinct primes of length $k$.

Let $N$ be the set of the natural numbers, $x \in N, Z_{x}^{*}=\{y \mid 1 \leq y<x, \operatorname{gcd}(x, y)=$ $1\}$ and $Z_{x}^{+1}=\left\{y \in Z_{x}^{*} \mid(y \mid x)=+1\right\}$, where $(y \mid x)$ is the jacobi symbol. We say that $y \in Z_{x}^{*}$ is a quadratic residue modulo $x$ iff there is $w \in Z_{x}^{*}$ such that $w^{2} \equiv y \bmod x$. If this is not the case we call $w$ a quadratic non residue modulo $x$.

Define the quadratic residuosity predicate to be

$$
Q_{x}(y)= \begin{cases}0, & \text { if } y \text { is a quadratic residue modulo } x \\ 1, & \text { otherwise }\end{cases}
$$

and the languages $Q R$ and QNR as

$$
\begin{gathered}
Q R=\left\{(y, x) \mid Q_{x}(y)=0\right\} \\
Q N R=\left\{(y, x) \mid y \in Z_{x}^{+1} \text { and } Q_{x}(y)=1\right\} .
\end{gathered}
$$


Fact 1: Let $\sim$ be the relation so defined: $y_{1} \sim y_{2}$ iff $Q_{x}\left(y_{1} y_{2}\right)=0$. Then $\sim$ is an equivalence relation in $Z_{x}^{+1}$. Two elements are equivalents if they have the same quadratic character modulo each of the prime divisors of $x$. Thus, if $x \in Z_{2}(k)$ there are 2 equivalence classes, if $x \in Z_{3}(k)$ there are 4 ; in general if $x=p_{i}^{h_{1}} \cdots, p_{n}^{h_{n}}$ where each $p_{i}$ is a prime $>2$ and $p_{i} \neq p_{i}$ if $i \neq j$, then there are $2^{n}$ equivalence classes.

Fact 2: For each $y_{1}, y_{2} \in Z_{x}^{+1}$ one has

$$
Q_{x}\left(y_{1} y_{2}\right)=Q_{x}\left(y_{1}\right) \oplus Q_{x}\left(y_{2}\right) .
$$

Fact 3: Where " $\oplus$ " denotes the exclusive or operator. the jacobi symbol function $x \mid n$ is polynomial-time computable.

We now formalize the complexity assumption that is sufficient for non-interactive zero-knowledge. Namely, that it is computationally hard to distinguish the integers product of 2 primes leftarrow the ones product of 3 primes.

\subsection{A Complexity Assumption}

2OR3A: for each poly-size family of circuits $\left\{C_{k} \mid k \in N\right\}$

$$
\left|P_{Z_{2}(k)}-P_{Z_{3}(k)}\right|<k^{-c}
$$

for all positive constants $c$ and sufficiently large $k$; where

$$
\begin{aligned}
& P_{Z_{2}(k)}=P_{R}\left(x \leftarrow Z_{2}(k): C_{k}(x)=1\right) \text { and } \\
& P_{Z_{3}(k)}=P_{R}\left(x \leftarrow Z_{3}(k): C_{k}(x)=1\right) .
\end{aligned}
$$

2OR3A is a stronger assumption than assuming that deciding quadratic residuosity is hard. (Having an oracle for $Q_{n}(\cdot)$, allows one to prbabilistically count the number of $\sim$ equivalence in $Z_{n}^{+1}$ and thus, by fact 1 , to distinguish whether $n \in Z_{2}(k)$ or $\left.n \in Z_{3}(k)\right)$. Thus we can freely use that quadratic residuosity is computationally hard (as formalized below) without increasing our assumption set.

Quadratic Residuosity Assumption(QRA):

For each poly-size family of circuits $\left\{C_{k} \mid k \in \mathcal{N}\right\}$,

$$
\begin{array}{r}
\operatorname{Pr}\left(x \leftarrow Z_{2}(k) ; y \leftarrow Z_{x}^{+1}: C_{k}(x, y)=Q_{x}(y)\right) \\
<1 / 2+1 / k^{-O(1)} .
\end{array}
$$

The QRA was introduced in [GM] and is now widely used in Cryptography. The current fastest algorithm to compute $Q_{x}(y)$ is to first factor $x$ and then compute $Q_{x}(y)$, while it is well known that, given the factorization of $x, Q_{x}(y)$ can be computed in $O\left(|x|^{3}\right)$ steps. In what follows, we choose $x \in Z_{2}(k)$ since these integers constitute the hardest input for any known factoring algorithm. 


\section{Single-Theorem Non-Interactive Zero-Knowledge Proofs}

To prove the existence of single-theorem Non-Interactive Zero-Knowledge Proof Systems (single-theorem non-interactive ZKPS) for all NP languages, it is enough to prove it for $3 C O L$ the NP-complete language of the 3-colorable graphs [GJ]. For $k>0$, we define the language $3 C O L_{k}=\{x \in 3 C O L|| x \mid \leq k\}$.

Definition 3.1. A Single-Theorem Non-Interactive ZKPS is a pair $(A, B)$ where $A$ (the Prover) is a Probabilistic Turing Machine and $B(\cdot, \cdot, \cdot)$ (the Verifier) is a deterministic algorithm running in time polynomial in the length of its first input, such that:

1. Completeness. (The probability of succeeding in proving a true theorem is overwhelming.)

$\exists c>0$ such that $\forall x \in 3 C O L_{k}$

$$
\begin{array}{r}
\operatorname{Pr}\left(\sigma \leftarrow\{0,1\}^{n^{c}} ; y \leftarrow A(\sigma, x):\right. \\
\quad B(x, y, \sigma)=1)>1-n^{-O(1)} .
\end{array}
$$

2. Soundness. (The probability of succeeding in proving a false theorem is negligible.)

$\exists c>0$ such that $\forall x \notin 3 C O L_{k}$ and for each Probabilistic Turing Machine $A^{\prime}$

$$
\begin{array}{r}
\operatorname{Pr}\left(\sigma \leftarrow\{0,1\}^{n^{c}} ; y \leftarrow A^{\prime}(\sigma, x):\right. \\
B(x, y, \sigma)=1)<n^{-O(1)} .
\end{array}
$$

3. Zero-Knowledge. (The proof gives no information but the validity of the theorem.)

$\exists c>0$ such that the family of random variables $V=\{V(x)\}$ is approximable over $3 C O L$. Where

$$
V(x)=\left\{\sigma \leftarrow\{0,1\}^{|x|^{c}} ; y \leftarrow A(\sigma, x):(\sigma, y)\right\},
$$

Remark: Notice that, as usual, the zero-knowledge condition guarantees that the verifier's view can be well simulated; that is, all the verifier may see can be reconstructed with essentially the same odds. In our scenario, what the verifier sees is only the common random string and the proof, i.e., the string, received by A. Notice that in our scenario, the definition of zero-knowledge is simpler. As there is no interaction between $B$ and $A$, we do not have to worry about possible cheating by the verifier to obtain a "more interesting view." That is, we can eliminate the quantification " $\forall B^{\prime \prime}$ from the original definition of [GMR]. 
Theorem 3.1 . Under the QRA, there exists a Single-Theorem Non-Interactive ZKPS for 3-COL.

This theorem will be rigorously proven in the final paper. Here we restrict ourselves to informally describe the programs $\mathrm{P}$ and $\mathrm{V}$ of a single-theorem non-interactive ZKPS $(\mathrm{P}, \mathrm{V})$ and, even more informally, to argue that they posses the desired properties.

\subsection{The Proof System (P,V)}

\section{Instructions for $P$}

1. Randomly select $n_{1}, n_{2}, n_{3} \in Z_{2}(k)$

2. For $i=1,2,3$ randomly select $q_{i}$ such that $\left(q_{i} \mid n_{i}\right)=1$ and $q_{i}$ is a quadratic non-residue $\bmod n_{i}$.

3. Color $G$ with colors $1,2,3$.

4. For each node $v$ of $G$ whose color is $i$, label $v$ with a randomly selected triplet $\left(v_{1}, v_{2}, v_{3}\right) \in Z_{n_{1}}^{+1} \times Z_{n_{2}}^{+1} \times Z_{n_{3}}^{+1}$ such that $Q_{n}\left(v_{i}\right)=0$ and $Q_{n_{j}}\left(v_{j}\right)=1$ for $j \neq i$. Call $G^{\prime}$ the so labeled $G$

\{Remark 1: WLOG (else purge $\sigma$ in the "right way") let $\sigma=\sigma_{1} \circ \sigma_{2} \circ \sigma_{3} \circ \sigma_{4}, \cdots$, where all triplets $\left(\sigma_{1}, \sigma_{2}, \sigma_{3}\right)\left(\sigma_{4}, \sigma_{5}, \sigma_{6}\right), \cdots$ belong to $\left.Z_{n_{i}}^{+1} \times Z_{n_{i}}^{+1} \times Z_{n_{3}}^{+1}\right\}$

\{Convention: The first $8 \dot{k}$ triplets are assigned to the first edge of $G$ (in the lexicographic order), the next $8 k$ triplets to the second edge, and so on. $\}$

5. For each edge $(a, b)$ of $G^{\prime}$ (where node $a$ has label $\left(a_{1}, a_{2}, a_{3}\right)$ and node $b$ $\left.\left(b_{1}, b_{2}, b_{3}\right)\right)$ and each of its $8 k$ assigned triplets $\left(z_{1}, z_{2}, z_{3}\right)$ compute one of the following types of signature.

(Comment: Only one is applicable if steps 1-4 are performed correctly)\}

$$
\begin{array}{ll}
\left(\sqrt{z_{1}}, \sqrt{z_{2}}, \sqrt{z_{3}}\right) & \text { type } 0 \\
\left(\sqrt{q_{1} z_{1}}, \sqrt{z_{2}}, \sqrt{z_{3}}\right) & \text { type } 1 \\
\left(\sqrt{z_{1}}, \sqrt{q_{2} z_{2}}, \sqrt{z_{3}}\right) . & \text { type } 2 \\
\left(\sqrt{z_{1}}, \sqrt{z_{1}}, \sqrt{q_{3} z_{3}}\right) & \text { type } 3 \\
\left(\sqrt{a_{1} z_{1}}, \sqrt{a_{2} z_{2}}, \sqrt{a_{3} z_{3}}\right) & \text { type } 4 \\
\left(\sqrt{b_{1} z_{1}}, \sqrt{b_{2} z_{2}}, \sqrt{b_{3} z_{3}}\right) & \text { type } 5 \\
\left(\sqrt{a_{1} b_{1} z_{1}}, \sqrt{a_{2} b_{2} z_{2}}, \sqrt{a_{3} b_{3} z_{3}}\right) & \text { type } 6 \\
\left(\sqrt{q_{1} z_{1}}, \sqrt{q_{2} z_{2}}, \sqrt{q_{3} z_{3}}\right) & \text { type } 7
\end{array}
$$

\{Notation "by example": Let $z_{1}$ be a quadratic non residue $\bmod n_{1}, z_{2}$ a quadratic residue $\bmod n_{2}$, and $z_{3}$ is a quadratic residue $\bmod n_{2}$. Then the signature of the triplet $\left(z_{1}, z_{2}, z_{3}\right)$ a triplet of type $1:\left(\sqrt{q_{1} z_{1}}, \sqrt{z_{2}}, \sqrt{z_{3}}\right)$ where $\sqrt{q z_{1}}$ denotes a randomly selected square root of the quadratic residue $q_{1} \cdot z_{1}$ $\bmod n_{1} ;$ and for $i=2,3 \sqrt{z_{i}}$ denotes a randomly selected square root of $z_{i} \bmod$ $\left.n_{i}\right\}$ 
6. Send $\mathrm{V} n_{1}, n_{2}, n_{3}, q_{1}, q_{2}, q_{3}, G^{\prime}$, and the signature of the triplets composing $\sigma$. \{Comment: Note that the edges of $G^{\prime}$ are labelled with triples, not with colors!\}

\section{Instructions for $\mathrm{V}$}

1. Verify that $n_{1}, n_{2}$, and $n_{3}$ are not even and not integer powers. Verify that $G^{\prime}$ is a proper labelling of $G$. That is, each node $v$ has assigned a triplet $\left(v_{1}, v_{2}, v_{3}\right)$ such that $v_{i} \in Z_{n_{i}}^{+1}$ for $i=1,2,3$.

2. Break $\sigma$ into triplets, verify that for each edge you received a signature of some type for each of its $8 k$ triplets.

3. If all the above verifications have been successfully made, accept that $G$ is 3-colorable.

\subsection{A Rough Idea of why $(P, V)$ is a Single-Theorem Non- Interactive ZKPS}

First notice that the communication is mono-directional: From $\mathrm{P}$ to $\mathrm{V}$. Then let us convince ourselves that the statement of Remark 1 really holds without loss of generality. In our context, WLOG means with overwhelming probability.

If $G$ has $a$ edges, our protocol assumes $\sigma$ to consist of $8 \cdot k \cdot a$ triplets in $Z_{n_{1}}^{+1} \times$ $Z_{n_{2}}^{+1} \times Z_{n_{3}}^{+1}$. Such a string $\sigma$ is easily obtainable from a (not too much larger) random string $\rho$. Consider $\rho$ to be the concatentation of $k$-bit strings grouped into triplets

$$
\rho=\left(\rho_{1}, \rho_{2}, \rho_{3}\right)\left(\rho_{4}, \rho_{5}, \rho_{6}\right) \cdots
$$

Then obtain $\sigma$ by "purging" $\rho$. That is, obtain $\sigma$ from $\rho$ by discarding all triplets not in $Z_{n_{1}}^{+1} \times Z_{n_{2}}^{+1} \times Z_{n_{3}}^{+1}$. We now argue that $\rho$ is not much longer than $\sigma$. Let $n$ be either $n_{1}$ or $n_{2}$ or $n_{3}$. Now a random $k$-bit integer (with possible leading 0 's) is less than $n$ with probability $\geq \frac{1}{2}$; a random integer less than $n$ belongs to $Z_{n}^{*}$ with probability $\geq \frac{1}{2}$; a random element of $Z_{n}^{*}$ belongs to $Z_{n}^{+1}$ with probability $\geq \frac{1}{2}$. Thus, we expect that at least 1 in 64 of the triplets of $\rho$ not to be discarded.

Now let us consider the question of V's running time. $V$ can verify in poly-time whether $n_{i}=x^{\alpha}$ (where $x, \alpha$ integers; $\alpha>1$ ) as only values $1, \cdots, \log n_{i}$ should be tried for $\alpha$ and binary search can be performed for finding $x$, if it exists. All other steps of $V$ are even easier.

Now let us give some indication that $(\mathrm{P}, \mathrm{V})$ constitute a single-theorem noninteractive ZKPS.

Completeness: Assuming that $\sigma$ is already consiting of triplets in $Z_{n_{1}}^{+1} \times Z_{n_{2}}^{+1} \times$ $Z_{n_{3}}^{+1}$, if $\mathrm{P}$ operates correctly, $\mathrm{V}$ will be satisfied with probability 1 .

Soundness: If the verification step 1 is successfully passed, by fact 1 , there must be $\geq 2 \sim$ equivalence classes in each $Z_{n_{i}}^{+1}$ (exactly two if $P$ honestly chooses all the $n_{i}$ 's in $Z_{2}(k)$ ). 
Thus, if we define two of our triplets $\left(z_{1}, z_{2}, z_{3}\right)\left(w_{1}, w_{2}, w_{3}\right)$ to be equivalent if $z_{i} w_{i} \bmod n_{i}$ is a quadratic residue for $i=1,2,3$, we obtain $\geq 8$ equivalence classes among the triplets (exactly 8 if $\mathrm{P}$ is honest).

To exhibit a signature of a given type for a triplet, essentially means to put the triplet in one out of $\leq 8$ possible "drawers". (there are 8 types of signatues, but they may not be mutually exclusive; thus two drawers may be equal). Moreover, it is easy to see that if two triplets are put in the same drawer, they must belong to the same equivalence class.

As $\sigma$ is randomly selected, each of its triplets in $Z_{n_{i}}^{+1} \times Z_{n_{2}}^{+1} \times Z_{n_{3}}^{+1}$ is equally likely to belong to any of the $\geq 8$ equally-numerous equivalence classes. However, since if there were $>8$ classes, there would be (by fact 1 ) at least 16 , the fact that all triplets can be fit in $\leq 8$ drawers, "probabilistically proves" several facts:

1. There are exactly 8 equivalence classes among the triplets and exactly 8 distinct drawers.

2. The $n_{i}$ 's are product of two distinct prime powers.

3. $Q_{n_{1}}\left(q_{1}\right)=Q_{n_{2}}\left(q_{2}\right)=Q_{n_{3}}\left(q_{3}\right)=1$

4. $Q_{n_{1}}\left(q_{1}\right)+Q_{n_{2}}\left(q_{2}\right)+Q_{n_{3}}\left(q_{3}\right)=2$

That is, $\left(a_{1}, a_{2}, a_{3}\right)$ is a proper color (i.e., properly encodes a color: Either 1,2 , or 3 ).

5. That $\left(b_{1}, b_{2}, b_{3}\right)$ is a proper color.

6. That $\left(a_{1}, a_{2}, a_{3}\right)$ and $\left(b_{1}, b_{2}, b_{3}\right)$ are different colors. Else drawer 6 and drawer 0 would be the same.

Item 6 being true for all edges in $G^{\prime}$ implies that $G$ is 3-colorable which is what was to be proven.

\section{Zero-Knowledgeness}

Let us specify the simulating machine $M$ that, under the QRA, generates a pair ( $\sigma$, proof) with the "right odds" on input $G$ (without any coloring!)

\section{Instructions for $M$}

1. Randomly select $n_{1}, n_{2}, n_{3}, \in Z_{2}(k)$ together with their prime factorization.

2. Randomly select $q_{1}, q_{2}, q_{3}$ so that $Q_{n_{1}}\left(q_{1}\right)=Q_{n_{1}}\left(q_{2}\right)=Q_{n_{3}}\left(q_{3}\right)=0$

3. For each node $v$ of $G$, label $v$ with a triplet $\left(v_{1}, v_{2}, v_{3}\right) \in Z_{n_{1}}^{*} \times Z_{n_{2}}^{*} \times Z_{n_{3}}^{*}$ such that $Q_{N_{1}}\left(v_{1}\right)=Q_{n_{2}}\left(v_{2}\right)=Q_{n_{3}}\left(v_{3}\right)=0$. Call $G^{\prime}$ the so labelled graph.

4. Construct $\sigma=\left(\sigma_{1}, \sigma_{2}, \sigma_{3}\right)\left(\sigma_{4}, \sigma_{5}, \sigma_{6}\right) \cdots$, such that each triplet $\left(\sigma_{3 j+1}, \sigma_{3 j+2}, \sigma_{3 j+3}\right)$ is randomly selected so that $Q_{n_{i}}\left(\sigma_{3 j+i}\right)=0$ for $i=1,2,3$.

\{Remark: Also in the simulation we only deal with already "purged strings". It is not hard to see that $M$ could also handle generating "unpurged strings". 
5. For each edge $(a, b)$ of $G^{\prime}$ and each of its assigned $8 k$ triplets $\left(z_{1}, z_{2}, z_{3}\right)$, choose an integer $i$ at random between 0 and 7 , and compute a signature of type $i$.

\{Comment: By using the prime factorization of the $n_{i}$.

6. Output $\sigma, n_{1}, n_{2}, n_{3}, q_{1}, q_{2}, q_{3}, G^{\prime}$, and the computed signatures.

We now informally argue that $M$ is a good simulator for the view of $\mathrm{V}$. Essentially, this is so because efficiently detecting that the triplets of $\sigma$ are not randomly and independently drawn from the space $Z_{n_{i}}^{+1} \times Z_{n_{2}}^{+1} \times Z_{n_{3}}^{+1}$ is tantamount as violating the QRA (to be explained in the final paper). For the same reason, it cannot be detected efficiently that $G^{\prime}$ is an illegal labelling or that $q_{1}, q_{2}, q_{3}$ are squares mod, respectively, $n_{1}, n_{2}, n_{3}$. Given that, the distribution of the various types of signature looks "perfect".

\{Remark: the reader is encouraged to verify that if $(P, V)$ uses part of the used $\sigma$ to show that another graph is 3 -colorable, then extra knowledge would leek. For instance that there exists 3 -coloring of $G$ and $H$ in which nodes $v_{1}$ and $v_{2}$ in $H$ respectively have the same clolors as nodes $w_{1}$ and $w_{2}$ in $G$.

\section{Security Against Chosen Ciphertext Attack}

One of the most beautiful gifts of complexity-based cryptography is the notion of a public-key cryptosystem. As proposed by Diffie and Hellman [DH], each user $U$ publicizes a string $P_{U}$ and keeps secret an associated string $S_{U}$. Another user, to secretely send a message $m$ to $U$, computes $y=E\left(P_{U}, m\right)$ and sends $y$; upon receiving $y, U$ retrieves $m$ by computing $D\left(S_{U}, y\right)$; here $E$ and $D$ are polynomial-time algorithms chosen so that it will be infeasible, for any other user, to compute $m$ from $y$.

Notice that in this set-up any other user is thought to be a "passive" adversary who tries to retrieve $m$ by computing solely on inputs $y$ and $P_{U}$. This is indeed a mild type of adversary and other types of attacks have been considered in the literature. It is widely believed that the strongest type of attack among all the natural ones is the chosen-ciphertext attack. In such an attack, someone tries to break the system by asking and receiving decryptions of ciphertexts of his choices. Rivest has shown that Rabin's scheme (whose breaking is, for a passive adversary, as hard as factoring if the messages are uniformily selected strings of a given length) is easily vulnerable to such an attack. Indeed, this is an attack feasible to any employee who works at the decoding equipment of, say, a large bank. The power by this attack is very well exemplified by an elegant scheme of Rabin [R] that is as secure as factoring (if the messages are uniformily selected strings of a given length) in the passive adversary model but is easily broken by chosen-ciphertext attack. Since observing this phenomenon, people tried to design cryptosystems invulnerable to such attacks, but in vain. A positive answer has been found [GMT] only allowing interaction, during the encryption process, between legal sender and legal receiver. However, for the standard (non-interactive) Diffie-and-Hellman model, the existence of a cryptosystem invulnerable to chosen ciphertext attack has been an open problem since 1978 . 
Non-interactive zero-knowledge proofs allow us to finally solve this problem. The essence of our solution (instead of its details) is informally described as follows. Instead of sending $U$ an encryption, $y$, of a message $m$, one is required to send two strings: $y$ and and $\sigma$, where $\sigma$ is a zero-knowledge and non-interactive proof that the sender knows the decoding of $y$. The "decoding equipment" (read: the decoding function) checks that $\sigma$ is convincing and, if so, outputs $m$, the decoding of $y$; Otherwise, it outputs nothing. Notice that, now, being able to use the decoding equipment provably is of no advantage! In fact, only when we feed it with ciphertexts whose decoding we can prove we know, does the decoding equipment output these decodings! In other words, the decoding equipment can only be used to output what we already know. A detailed discussion of this powerful application will appear in the final paper.

(A formal setting and the proof require some care. For instance, the decoding equipment may be used as an oracle to check whether a given string $\sigma$ is a "correct proof of knowledge". Thus, in particular, one should prove that such an oracle cannot help. In the final paper we will essentially show that if one can generate a legal $(y, \sigma)$ pair without having $m$ as an input, then one can easily decrypt all messages on input $y$ and $P_{U}$ only.)

\section{References}

[ACGS] W. Alexi, B. Chor, O. Goldreich, and C. Schnorr RSA/Rabin Bits Are 1/2+ $1 /$ poly $(\log N)$ Secure, To appear SIAM J. on Computing.

[B1] M. Blum, Coin Flipping by Telephone, IEEE COMPCON 1982, pp. 133-137.

[B2] M. Blum, unpublished manuscript

[BBS] M. Blum, L. Blum and M. Shub, $A$ simple and secure pseudo-randomnumber generator,SIAM Journal of Computing, 1986

[BFM] Blum, De Santis, Feldman, Micali, and Persiano, Non-Interactive Zero Knowledge and Its Applications, in preparation.

[BGGHKMR] M. Ben-Or, O. Goldreich, S. Goldwasser, J. Hastad, J. Kilian, S. Micali, and P. Rogaway, Everything Provable is Provable in Zero-Knowledge, These Proceedings

[BH] R. Boppana, J. Hastad and S. Zachos, Interactive Proofs Systems for CO-NP Imply Polynomial Time Hierarchy Collapse, In preperation.

[BM] M. Blum and S. Micali, How To Generate Sequences Of Cryptographically Strong Pseudo-Random Bits, SIAM J. on Computing, Vol. 13, Nov 1984, pp. 850864

[DH] Diffie, W., and M.E. Hellman, New Directions in Cryptography,IEEE Trans. on Inform. Theory,

[DMP] De Sentis, Micali, and Persiano, Non Interactive Zero-Knowledge Proof Systems, Proc. Crypto 87.

[F] L. Fortnow, The Complexity of Perfect Zero-Knowledge, Proc. 19th ann. Symp. on Theory of Computing, New York, 1987.

[FFS] Feige, Fiat and A. Shamir, Zero-knowledge proofs of identity, Proceedings of the 19th Annual ACM Symp. on Theory of Computing, 1987, pp. 210-217 
[GM] S. Goldwasser, and S. Micali, Probabilistic Encryption, JCSS Vol. 28, No. 2, April 1984.

[GMR] S. Goldwasser, S. Micali and C. Rackoff, The Knowledge Complexity of Interactive Proof-Systems, To appear SIAM J. on Computing (manuscript available from authors).

[GoMiRi] S. Goldwasser, S. Micali, and R. Rivest, A Digital Signature Scheme Secure Against Adaptive, Chosen Cyphertext Attack To appear in SIAM J. on Computing (available from authors)

[GMT] S. Goldwasser, S. Micali, and P. Tong, Why and how to establish a perivate code in a public network, Proc. 23rd Symp. on Foundations of Computer Science, Chicago, Ill., 1982

[GMW] O. Goldreich, S. Micali and A. Wigderson, Proofs that Yield Nothing but their Validity and a Methodology of Cryptographic Design, Proc. of FOCS 1986.

[GMW2] O.Goldreich, S. Micali and A. Wigderson, How to Play Any Mental Game, Proceedings of the 19th Annual ACM Symp. on Theory of Computing, 1987, pp. 218-229.

[GS] S. Goldwasser and M. Sipser, Private Coins versus Public Coins in Interactive Proof Systems, Proceedings of the 18th Annual ACM Sympl on Theory of Computing, 1986, pp. 59-68.

[I] R. Impagliazzo, Personal Communication.

[MS] S. Micali and A. Shamir An improvement of the Fiat-Shamir Identification and Signature Scheme, These proceedings

[R] M. Rabin, Digitalized signatures and public-key functions as intractable as factorization, MIT/LCS/TR-212, Technical report MIT, 1978

[Y] A.Yao, Theory and Application of Trapdoor Functions, Proc. of 23rd FOCS, IEEE, Nov., 1982, pp. 80-91. 